\title{
Tamoxifen Regimen
}

National Cancer Institute

\section{Source}

National Cancer Institute. Tamoxifen Regimen. NCI Thesaurus. Code C160121.

A hormone therapy regimen consisting of tamoxifen that may be used in the treatment of breast cancer. 